\title{
Hydroxyapatite Formation of Dental Restorative Materials
}

\author{
Tran D*, Kang Y, Dunn K and Kugel G \\ Tufts University School of Dental Medicine, USA
}

\begin{abstract}
*Corresponding author: Duong Tran, DDS, MPH, PhD, Tufts University School of Dental Medicine, 1 Kneeland Street, Boston, MA, 02111, USA, Tel: 617636 3614; Fax: 617636 6511; Email: Duong.tran@tufts.edu
\end{abstract}

\section{Research Article}

Volume 5 Issue 3

Received Date: July 09, 2020

Published Date: July 20, 2020

DOI: $10.23880 /$ oajds- 16000263

\section{Abstract}

Objective: This study aimed to analyze the formation of hydroxyapatite (HA) of dental restorative materials.

Materials and Methods: The bioactivity of four materials were studied in vitro: Cention N (Ivoclar Vivadent) (Cention), ACTIVA $^{\text {TM }}$ BioACTIVE-RESTORATIVE ${ }^{T M}$ (Pulpdent) (Activa), Filtek ${ }^{T M}$ Bulk Fill Flowable Restorative (3M) (Filtek), and Fuji IX GP ${ }^{\circledR}$ (Fuji). Disc-shaped specimens of each material $(\mathrm{N}=12), 4 \mathrm{~mm}$ thick and $5 \mathrm{~mm}$ in diameter, were created by placing the materials in a mold and cured following the manufacturers' instructions. The specimens were polished and stored in individual $20 \mathrm{~mL}$ Falcon-tubes containing $10 \mathrm{~mL}$ phosphate buffered saline (PBS), five of each group for 2 weeks and other five of each group for 7 weeks. Two specimens of each material served as the control samples and stored in distilled water. The PBS solution was replaced every week. The specimens were then removed and washed thoroughly with distilled water and dried at room temperature. Amray 3300 FESEM (SEMTech) field scanning electron microscope (SEM) imaging and PulseTor SDD (Silicon Drift Detector) Energy-dispersive X-ray spectroscopy (EDS) analysis were used to determine the surface characteristics (morphology) and chemical composition of the surface precipitate. Weight percentage of calcium and phosphorous were compared between the control, 2- and 7-weeks groups for each material, and between materials for control, 2, and 7 weeks groups.

Results: Cention displayed significantly higher weight percentage of surface precipitate calcium (19.6\% at 2 weeks and 20.9 $\%$ at 7 weeks) and phosphorous ( $15.8 \%$ at 2 weeks and $14.5 \%$ at 7 weeks) compared to controls and other materials. SEM images showed agglomerates of spherical particles on the surface of Cention.

Conclusion: All results indicated that HA was formed on the surfaces of Cention specimens stored in PBS.

Keywords: Hydroxyapatite; Restorative material; Bioactivity; Scanning electron microscope

Abbreviations: SEM: Scanning Electron Microscope; EDS: Energy Dispersive X-ray Spectroscopy; PBS: Phosphate Buffered Saline; HA: Hydroxyapatite; IRM: Intermediate Restorative

\section{Introduction}

Modern dental practice has witnessed an increase in the use of bioactive dental materials to replace tooth structure [1-4]. Bioactive dental materials possess the ability to form apatite-like material on its surface when placed in a simulated body fluid for a period of time $[1,4,5]$. Apatite-like minerals deposition on the tooth structure and restoration interface is essential for re-mineralization and caries prevention. Several major bioactive restorative materials have been developed for dental practice that function through varying mechanisms of action [1,2]. Glass ionomers derivatives such as ACTIVA BioACTIVE-RESTORATIVE (Pulpdent) release calcium and phosphate allowing for the formation of hydroxyapatite (HA) [2]. Several bioactive dental materials developed for crown and bridge cementation such as Ceramir Crown \& Bridge (Doxa Dental AB) specifically deposit HA on the surfaces of samples stored in saliva as well as on samples stored in phosphate buffered saline (PBS) [6,7]. For endodontic 


\section{Open Access Journal of Dental Sciences}

treatment, there is a class of bioactive materials that have tissue regeneration capabilities, such as calcium phosphate cements, Alpha-BSM $\AA$, and calcium silicate cements, Mineral Trioxide Aggregate [8,9].

The formulations of conventional glass ionomers have been continuously changed to improve the strength, esthetics, and bioactivity [10,11]. Ivoclar's glass ionomer, Cention N, offers greater mechanical strength than glass ionomers, good handling and better esthetics than both amalgam and glass ionomers [12]. Regarding bioactivity, Cention N contains fluoroaluminosilicate glass which releases substantial levels of fluoride, hydroxide $(\mathrm{OH})$, and calcium $\left(\mathrm{Ca}^{2+}\right)$ ions into surrounding enamel and dentin. When the oral cavity's $\mathrm{pH}$ decreases due to acid produced by active cariogenic bacteria, Cention $\mathrm{N}$ releases a significant amount of ions which can help prevent demineralization of the tooth substrate. Increased availability of fluoride and calcium ions prevents enamel demineralization, promotes remineralization, and reduces bacterial plaque growth. Specifically, the alkaline glass of Cention $\mathrm{N}$ releases hydroxide ions which neutralize acid produced by cariogenic bacterial [13].

HA deposition on a bioactive material surface needs adequate concentration of necessary ions, adequate range of $\mathrm{pH}$, and a negatively charged surface [14-17]. Cention $\mathrm{N}^{\prime} \mathrm{s}$ alkaline filler can contribute fluoride, hydroxide $\left(\mathrm{OH}^{-}\right)$, and calcium $\left(\mathrm{Ca}^{2+}\right)$ ions. The combination of these ions with phosphate ions in saliva can essentially form HA. However, there has been a limited amount of research about HA formation capability of Cention $\mathrm{N}$ in comparison with other bioactive restorative materials. This study aimed to compare the formation of HA between Cention $\mathrm{N}$ (Cention) with three commonly used dental restorative materials, ACTIVA ${ }^{\mathrm{TM}}$ BioACTIVE-RESTORATIVE ${ }^{\mathrm{TM}}$ (Pulpdent) (Activa), Filtek ${ }^{\mathrm{TM}}$ Bulk Fill Flowable Restorative (3M) (Filtek), and Fuji IX GP® (Fuji).

\section{Materials and Methods}

\section{Samples Preparation}

The bioactivity of four restorative materials was studied in vitro. Cention $\mathrm{N}$ is a tooth-colored filling material for direct restorations of Class I, II or V. Its separate powder and liquid components are mixed by hand directly before use. The liquid contains dimethacrylates and inhibitors and accelerators, whilst the powder contains a barium aluminum silicate glass filler, ytterbium trifluoride, Isofiller (Tetric N-Ceram technology), a calcium barium aluminum fluorosilicate glass filler, a calcium fluorosilicate (alkaline) glass filler, initiators and pigments [13]. ACTIVA is a bioactive composite consisting of an ionic resin matrix, a shock-absorbing resin component and bioactive fillers. It releases and recharges with calcium, phosphate and fluoride ions [18]. These ions are essential element to form HA at the material-tooth interface. HA deposition at the interface connects the restoration and the tooth together, penetrates and fills micro-gaps, reduces sensitivity, guards against secondary caries, and seals margins against microleakage and failure. GC Fuji IX GP is a true glass ionomer which chemically bonds to tooth structure and releases significant levels of rechargeable fluoride. It is intended for geriatric and pediatric restorations, final restorations (non-stress areas), Intermediate Restorative (IRM), core material and long-term, temporary restorations. Filtek Bulk Fill Flowable Restorative is a composite used as a positive control group. It is a low viscosity, visible-light activated, radiopaque flowable composite containing zirconia/silica and ytterbium trifluoride filler of different sizes from 0.01 to $5.0 \mu$ [19].

48 disc-shaped specimens were prepared according to the manufacturer instructions. The materials were placed to a metal mold $4 \mathrm{~mm}$ thick by $5 \mathrm{~mm}$ diameter and covered by a glass slide on the top to remove any excess material and to flatten the upper surface. Cention and Fuji self-cured while Activa and Filtek were light cured (DEMI ${ }^{\mathrm{TM}}$, Kerr) for 40 seconds on each side. The specimens were removed after 10 minutes and wet-ground polished (EcoMet ${ }^{\mathrm{TM}} 250$, Buehler) sequentially from coarse to smooth grit with final polishing done at 600 grit polishing paper to achieve consistency in surface smoothness and glossiness. The specimens were placed in individual $20 \mathrm{~mL}$ Falcon-tubes containing 10 $\mathrm{mL}$ phosphate-buffered saline (PBS) so that both sides were exposed to the PBS. 5 specimens of each group were placed in the PBS for 2 weeks and other 5 specimens of each group were placed in the PBS for 7 weeks. The PBS solution were replaced every week. The specimens were then be removed and washed thoroughly with distilled water to remove adhering foreign ions on the surface and dried at room temperature. 2 specimens from each group served as a control and were placed in distilled water. The specimen surfaces were then be subjected to Amray 3300 FESEM (SEMTech) scanning electron microscopy (SEM) imaging and PulseTor SDD (Silicon Drift Detector) electron dispersive x-ray spectroscopy (EDS) analysis. The surface characteristics (morphology) and chemical composition of the surface precipitate, calcium phosphates were analyzed by EDS and were compared to that of the control specimens.

\section{Sample Size}

The statistical software Stata 15 was used for sample size calculation. Pilot testing reported that the deposited calcium levels of the precipitate layer were 27 weight percent (wt\%) for Cention N2 and 4.8 wt $\%$ for ACTIVA ${ }^{\text {TM }}$ BioACTIVERESTORATIVE $[13,20]$. Suppose that the standard deviations are 10 and $5 \mathrm{wt} \%$, respectively. To detect a mean difference 


\section{Open Access Journal of Dental Sciences}

of $22.2 \mathrm{wt} \%$ in the deposited calcium between two groups, 10 specimens ( $n=5$ per group) will be needed to achieve a power of $80 \%$ and significance level of 0.05 . The sample size for 2- and 7-week measurements will be 10 for each group. 2 specimens were used as negative control for each group. The total sample size for 4 groups was 48 specimens $(n=12$ per group).

\section{Data Analysis}

Descriptive statistics (means and standard deviations) were calculated for the concentration of the elements. Differences between concentration values across groups were determined using one-way ANOVA. Post-hoc pairwise tests were performed using Tukey's HSD test. P-values less than 0.05 was considered statistically significant. Stata 15 was used in the analysis.

\section{Results}

Cention displayed significantly higher weight percentage of surface precipitate calcium $(19.6 \%$ at 2 weeks and $20.9 \%$ at 7 weeks) and phosphorous (15.8\% at 2 weeks and $14.5 \%$ at 7 weeks) compared to controls and other materials (Tables $1 \& 2$, Figures 1 \& 2). There was no difference in the weight percentage of surface precipitate calcium and phosphorous between 2 weeks and 7 weeks for Cention, Filtek and Fuji. However, Activa showed a significant higher calcium and phosphorous deposition on the surface at 2 weeks compared to 7 weeks. SEM images showed agglomerates of spherical particles on the surface of Cention indicating HA formation (Figure 3). There were no agglomerates of spherical particles on the surface of Control, Activa, Filtek, and Fuji.

\begin{tabular}{|c|c|c|c|c|}
\hline Material & Control & 2 weeks & 7 weeks & P value \\
\hline Cention & $1.6(0.5)^{\mathrm{a}, \mathrm{b}}$ & $19.6(4.5)^{\mathrm{a}, \mathrm{h}, \mathrm{i}}$ & $20.9(5.9)^{\mathrm{b}, \mathrm{l}, \mathrm{m}}$ & 0.07 \\
\hline Activa & $2.3(0.1)^{\mathrm{c}, \mathrm{e}, \mathrm{f}}$ & $4.9(0.5)^{\mathrm{c}, \mathrm{d}, \mathrm{j}, \mathrm{k}}$ & $2.3(0.6)^{\mathrm{d}, \mathrm{n}, \mathrm{o}}$ & 0.02 \\
\hline Filtek & $0.1(0)^{\mathrm{e}}$ & $0.1(0.1)^{\mathrm{h}, \mathrm{j}}$ & $0.1(0.1)^{\mathrm{l}, \mathrm{n}}$ & 0.4 \\
\hline Fuji & $0.1(0)^{\mathrm{f}}$ & $0.2(0.1)^{\mathrm{i}, \mathrm{k}}$ & $0.1(0.04)^{\mathrm{m}, \mathrm{o}}$ & 0.6 \\
\hline P value & 0.1 & 0.001 & 0.001 & \\
\hline
\end{tabular}

Values with the same superscript letter in the same row or column indicated a significant difference between two groups.

Table 1: Weight (grams) \% of Calcium; standard deviations are given in parenthesis.

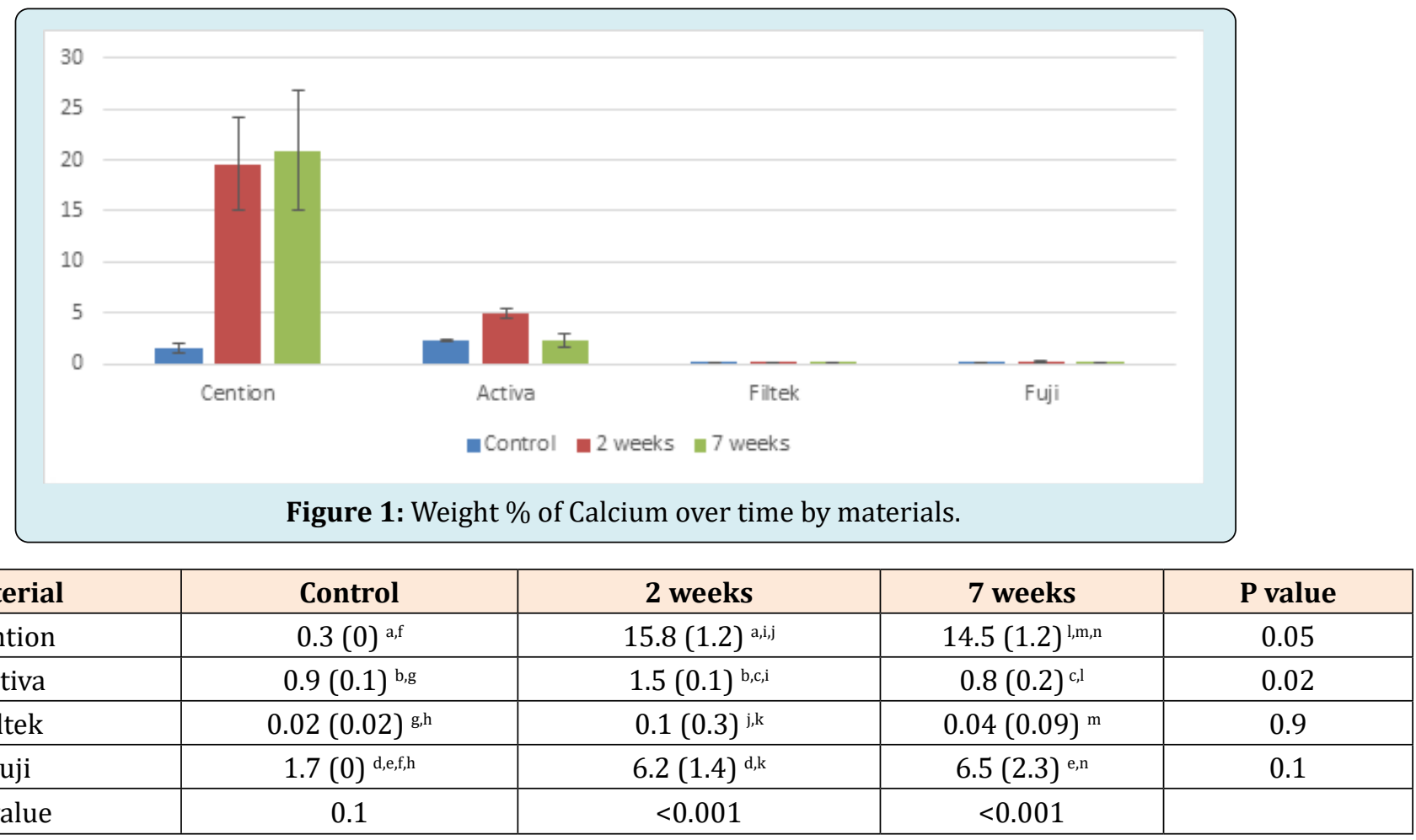

Values with the same superscript letter indicated significant difference between two groups.

Table 2: Weight (grams) \% of Phosphorous; standard deviations are given in parenthesis. 


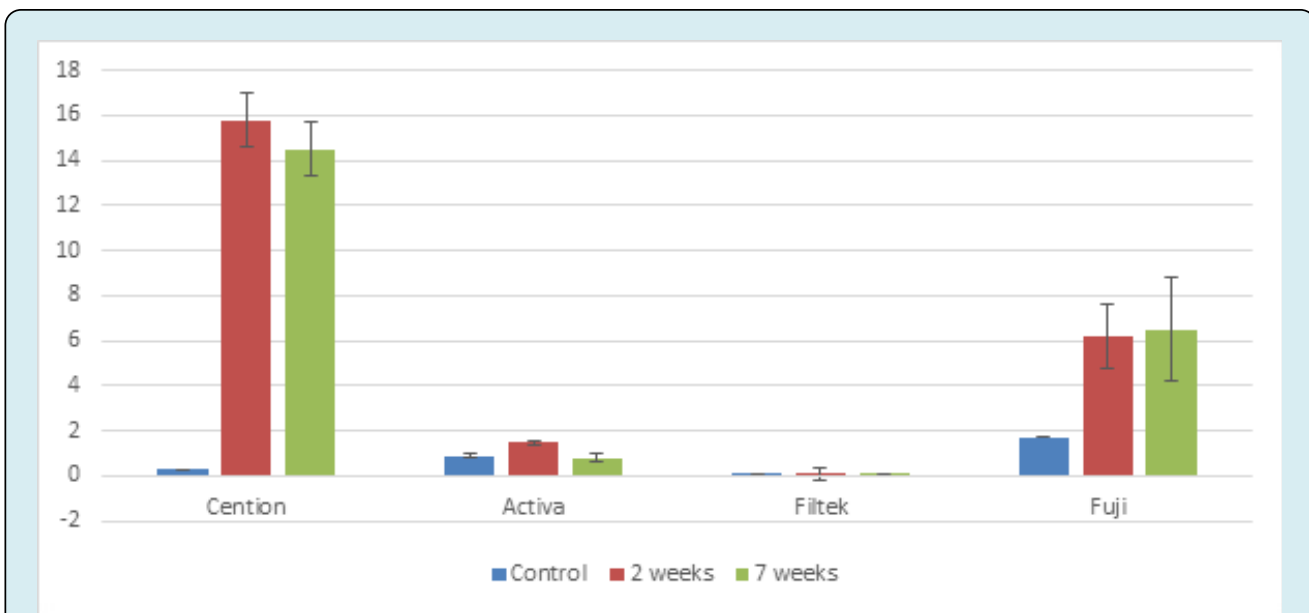

Figure 2: Weight \% of Phosphorous over time by materials.

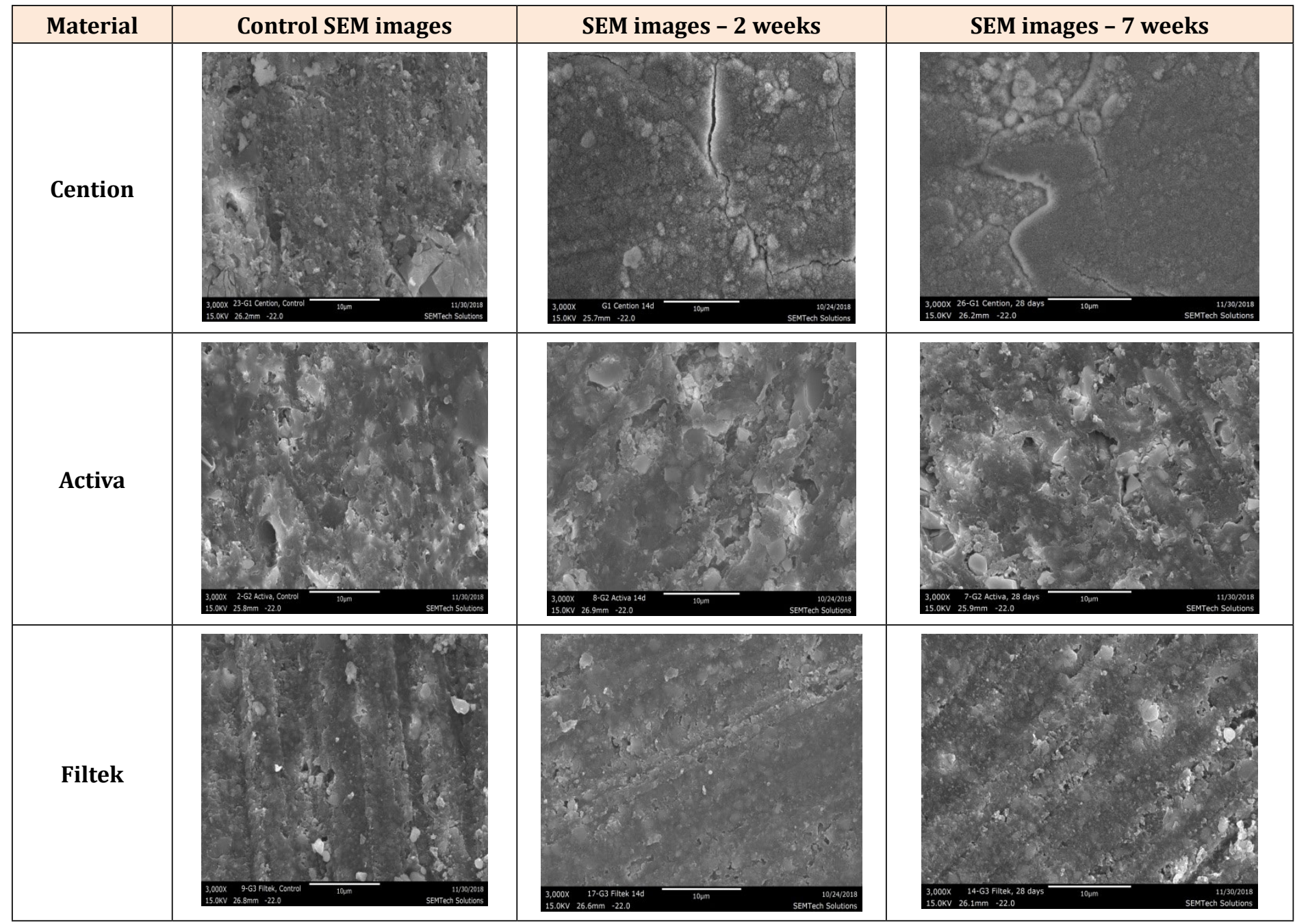




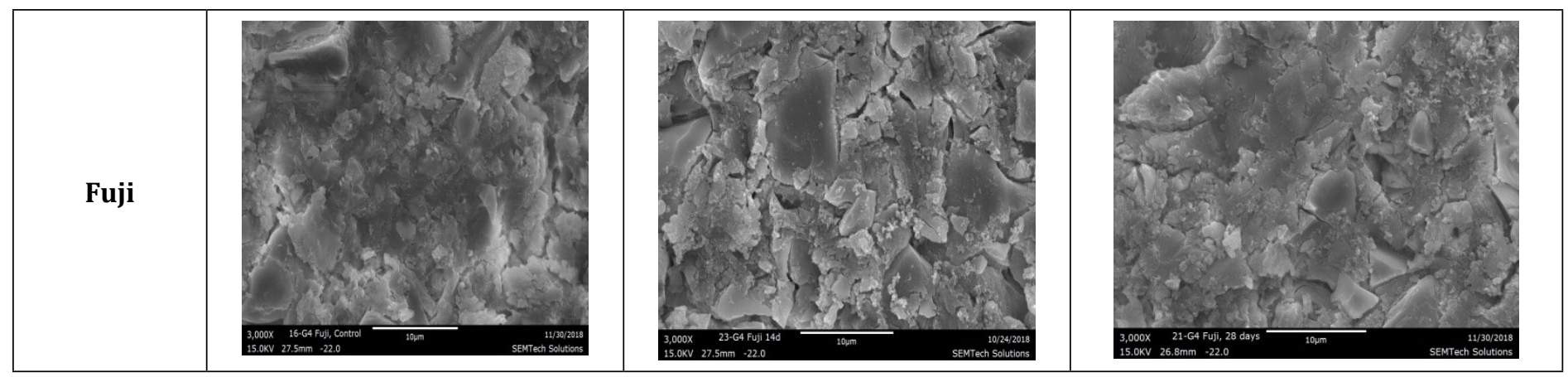

Figure 3: SEM images of specimens.

\section{Discussion}

SEM images of Cention demonstrated HA formation in the form of spherical particles on the surface at $2 \& 7$ weeks while SEM images of Control, Activa, Filtek, and Fujidid not show any spherical particles or HA formation. The HA formation was supported by a significantly higher weight percentage of surface precipitate calcium $(19.6 \%$ at 2 weeks and $20.9 \%$ at 7 weeks) and phosphorous (15.8\% at 2 weeks and $14.5 \%$ at 7 weeks) for Cention compared to controls and other materials. Cention displayed a high concentration of $\mathrm{Ca} 2+$ and P042- deposition which was a prerequisite for for HA formation. $\mathrm{Ca} 2+$ and P042- saturate when their concentrations are above the solubility limit for HA. A study also showed that weight percentage of $\mathrm{Ca} 2+$ and P042- were $27 \%$ and $22 \%$, respectively, of the precipitate layer on the Cention surface after one month storage in an artificial saliva solution [13]. The $\mathrm{pH}$ of the PBS solution facilitates the HA formation because a $\mathrm{pH}$ range from 4.2 and 12 is favorable for HA stability $[17,21]$. Another prerequisite for HA formation is a negatively charged surface in order to achieve HA precipitation on a material surface [17]. $\mathrm{Ca}^{2+}$ ions first connect to a negatively charged surface to create a positively charged surface layer that in turn link the $\mathrm{PO}_{42-}$ in the PBS solution. In case of Cention, the setting reaction may create a negative surface charge, but this hypothesis needs to be further studies in future. In addition, the presence of $\mathrm{CaF}_{2}$ and $\mathrm{Ca}_{3}\left(\mathrm{PO}_{4}\right) 2$ on the surface of Cention specimens were also contributed to the $\mathrm{Ca}^{2+}$ and $\mathrm{PO}_{42-}$ ions release from the alkaline glass filler [13].

In our study, Activa, Fuji, and Filtek surface did not contain nearly as much $\mathrm{Ca}^{2+}$ and $\mathrm{PO}_{42-}$ compared to Cention. Their SEM images did not show any HA precipitation on the material surface either. A study demonstrated the long term calcium ion release at the neutral pH of 6.8 of Cention, Fuji IX GP Fast, and Fuji II [13]. Of the three products, Cention $\mathrm{N}$ exhibited the highest calcium ion release after 180 days. Filtek is a composite which serve as a positive control group so we din't expect to see a release of high concentration of calcium and phosphate. Additionally, GIC is an acidic material due to its main components, polyacrylic acid, so its acidic condition may not favor HA formation.

The results clearly indicated that Cention promotes in vitro HA formation. However, it is unknown if the in vitro bioactivity remains significant due to different chemical environment in the mouth compared to the PBS used in this study. It will add more value to the study to show how deep the HA was formed under specimen surface and morphology of HA deposit. In addition, there is a biological contribution that is hard to simulate in vitro. Further study is needed to answer these questions.

\section{Conclusion}

HA was formed on the surfaces of Cention specimens when immersed in a in PBS, indicating in vitro bioactivity. In the meanwhile, the controls, Activa, and Fuji, did not show in vitro bioactivity.

\section{References}

1. Chen L, Shen H, Suh BI (2013) Bioactive dental restorative materials: a review. Am J Dent 26(4): 219-227.

2. Hamdy TM (2018) Bioactivity: A New Buzz in Dental Materials. EC Dental Science 17: 1278-1283.

3. Jefferies SR (2014) Bioactive and biomimetic restorative materials: a comprehensive review. Part I. J Esthet Restor Dent 26(1): 27-39.

4. Vallittu PK, Boccaccini AR, Hupa L, Watts DC (2018) Bioactive dental materials-Do they exist and what does bioactivity mean? Dent Mater 34(5): 693-694.

5. ISO Standard Proposal ISO/TC 150/SC 1 Part 1 and 2.

6. Engstrand J, Unosson E, Engqvist H (2011) A dental cement capable of calcium phosphate formation on its surface during water storage. European Cells and Materials 21(1): 35. 
7. Engstrand J, Unosson E, Engqvist H (2012) Hydroxyapatite formation on a novel dental cement in human saliva. ISRN dentistry.

8. Torabinejad M, Parirokh M, Dummer P (2018) Mineral trioxide aggregate and other bioactive endodontic cements: an updated overview-part II: other clinical applications and complications. Int Endod J 51(3): 284317.

9. O Neill R, McCarthy H, Montufar E, Ginebra MP, Wilson DI, et al. (2017) Critical review: Injectability of calcium phosphate pastes and cements. Acta biomaterialia 50: 1-19.

10. May E, Donly KJ (2017) Fluoride release and re-release from a bioactive restorative material. American Journal of Dentistry 30(6): 305-308.

11. Heck K, Frasheri I, Diegritz C, Manhart J, Hickel R, et al. (2020) Six-year results of a randomized controlled clinical trial of two glass ionomer cements in class II cavities. J Dent 97: 103333.

12. Jayaraj D, Simon EP, Kumar MR (2018) Cention N: A review. Dental Bites pp: 14.

13. Ivoclar Vivadent AG. Scientific Documentation: Cention N. https://asia.ivoclarvivadent.com/zoolu-website/ media/document/38546/Cention+N. Accessed June 22, 2020.
14. Kokubo T, Takadama H (2006) How useful is SBF in predicting in vivo bone bioactivity? Biomaterials 27(15): 2907-2915.

15. Kim HM (2003) Ceramic bioactivity and related biomimetic strategy. Current opinion in solid state and materials science 7(4-5): 289-299.

16. Kim HM, Himeno T, Kokubo T, Nakamura T (2005) Process and kinetics of bonelike apatite formation on sintered hydroxyapatite in a simulated body fluid. Biomaterials 26(21): 4366-4373.

17. Lööf J, Engqvist H, Gómez-Ortega G, Spengler H, Ahnfelt NO, et al. (2005) Mechanical property aspects of a biomineral based dental restorative system. Paper presented at: Key Engineering Materials.

18. Slowikowski L, John S, Finkleman M, Perry R, Harsono M, et al. (2014) Fluoride ion release and recharge over time in three restoratives. J Dent Res 93: 268.

19. ESPE M. Filtek ${ }^{\mathrm{TM}}$ Bulk Fill Flowable Restorative.

20. Chao W, Perry R, Kugel G (2016) Surface deposition analysis of bioactive restorative material and cement. J Dent Res 95.

21. Chow LC, Eanes ED (2001) Monographs in oral science, Switzerland: S. Karger AG, 18. 